\title{
A New Minimal Average Weight Representation for Left-to-Right Point Multiplication Methods
}

\author{
Majid Khabbazian, T. Aaron Gulliver, Senior Member, IEEE,, Vijay K. Bhargava, Fellow, IEEE
}

\begin{abstract}
This paper introduces a new radix-2 representation with the same average weight as the width- $w$ nonadjacent form ( $w$-NAF). In both $w$-NAF and the proposed representations, each nonzero digit is an odd integer with absolute value less than $M$. However, for $w$-NAF, $M$ is of the form $2^{w-1}$, while for the proposed representation it can be any positive integer. Therefore, using the proposed integer representation we can use the available memory efficiently, which is attractive for devices with limited memory. Another advantage of the proposed representation over $w$-NAF is that it can be obtained by scanning the bits from left-toright. This property is also useful for memory-constrained devices because it can reduce both the time and space complexity of fast point multiplication techniques.
\end{abstract}

Index Terms - minimum-weight representation, left-to-right recoding, efficient implementation, point multiplication, elliptic curve cryptosystems.

\section{INTRODUCTION}

In 1985 Koblitz [1] and Miller [2] proposed the group of points on an elliptic curve over a finite field to create the elliptic curve discrete logarithm problem (ECDLP). Unlike the discrete logarithm problem over the multiplicative group of a finite field (DLP), there is no known subexponential-time algorithm to solve the ECDLP in general. Thus, elliptic curves are an attractive alternative to implement many cryptographic techniques such as Diffie-Hellman [3] and ElGamal [4].

Point multiplication dominates the execution time of elliptic curve cryptosystems, so various methods have been studied to enhance the performance of this operation [5], [6]. Specifically, the integer representation of the multiplier plays an important role in the performance of these methods [7]. Among the existing integer representations, those with minimal average Hamming weight (number of nonzero digits) such as $w$ NAF are more attractive. This is due to the fact that they reduce the required number of point additions/subtractions. It is also of interest to have a representation which can be obtained by scanning the bits from left to right (i.e., from the most significant bit to the least significant bit) [8], [9]. This property eliminates the need for recoding and storing the multiplier in advance, hence improving the performance of left-to-right point multiplication methods in terms of running time and memory. We are also interested in representations which allow efficient usage of memory. This is important for devices with a small amount of memory. In [10], the author proposes a generalized $w$-NAF which allows more efficient

M. Khabbazian and V. K. Bhargava are with the Department of Electrical and Computer Engineering, University of British Columbia, Canada. E-mail: \{majidk, vijayb\}@ece.ubc.ca.

T. A. Gulliver is with the Department of Electrical and Computer Engineering, University of Victoria, Canada. E-mail: agullive@ece.uvic.ca. usage of memory than $w$-NAF. However, similar to the $w$ NAF recoding algorithm, the generalized $w$-NAF recoding algorithm scans the bits from right to left.

In this paper, we introduce a new integer representation which has the same average weight as $w$-NAF, but has the advantage that it results in a point multiplication method which uses less memory. This memory saving results from the fact that the new representation can be obtained by scanning the multiplier bits from left to right. It can also result in more efficient usage of memory. This is because in the left-to-right point multiplication algorithms (such as Algorithm 1, given in Section II-B), we need to store $m$ points where $m$ is the number of positive integers in the digit set. For $w$-NAF, the representations proposed in [11], [12] and $w$-MOF [13] ${ }^{1}, m$ is of the form $2^{w-2}$, where $w$ is a positive integer greater than 1. So if we have for example sufficient memory for storing seven points in a small device with little storage space, using these representations we can only store up to four points and the rest of the memory is wasted. However, using the proposed representation we are able to use all available memory to store seven points. This is due to the fact that for the proposed representation $m$ can be any positive integer.

The rest of this paper is organized as follows. In Section II, we briefly review elliptic curves. Section III proposes the new integer representation and presents an efficient recoding algorithm to generate it. In Section IV, we analyze the average Hamming weight of the proposed representation. In Section $\mathrm{V}$, we evaluate the memory requirements of the proposed recoding algorithm and then compare it with the previous algorithms. Finally, we give some applications of the proposed representation and the conclusions in Sections VI and VII, respectively.

\section{Elliptic CuRves}

An elliptic curve $E$ over the field $\mathbb{F}$ is a smooth curve in the so called "long weirestraß form"

$$
E: y^{2}+a_{1} x y+a_{3} y=x^{3}+a_{2} x^{2}+a_{4} x+a_{6},
$$

where $a_{1}, \ldots, a_{6} \in \mathbb{F}$. Equation (1) may be simplified to

$$
E: y^{2}=x^{3}+a x+b \text {, }
$$

and

$$
E: y^{2}+x y=x^{3}+a x^{2}+b,
$$

when $\operatorname{Char}(\mathbb{F}) \neq 2,3$ and $\operatorname{Char}(\mathbb{F})=2$, respectively.

\footnotetext{
${ }^{1}$ This paper appeared after our work was submitted and we only recently became aware of it.
} 


\section{A. Point Addition}

Let $E(\mathbb{F})$ be the set of points $P=(x, y) \in \mathbb{F}^{2}$ that satisfy the elliptic curve equation (along with a "point at infinity" denoted $\mathcal{O}$ ). It is well known that $E(\mathbb{F})$ together with the point addition operation given in Table I form an abelian group. As shown in Table II, point inversion in this group can be computed easily. Note that in both Tables I and II, the points $P_{1}=\left(x_{1}, y_{1}\right), P_{2}=\left(x_{2}, y_{2}\right)$ and $P_{3}=\left(x_{3}, y_{3}\right)$ are represented in affine coordinates.

\begin{tabular}{|l||l|l|}
\hline \multirow{4}{*}{$\operatorname{Char}(\mathbb{F})=2$} & $P_{1} \neq \pm P_{2}$ & $\begin{array}{l}\lambda=\frac{y_{2}+y_{1}}{x_{2}+x_{1}} \\
x_{3}=\lambda^{2}+\lambda+x_{1}+x_{2}+a \\
y_{3}=\left(x_{1}+x_{3}\right) \lambda+y_{1}+x_{3}\end{array}$ \\
\cline { 2 - 3 } & $P_{1}=P_{2}$ & $\begin{array}{l}\lambda=\frac{y_{1}}{x_{1}}+x_{1} \\
x_{3}=\lambda^{2}+\lambda+a \\
y_{3}=\left(x_{1}+x_{3}\right) \lambda+y_{1}+x_{3}\end{array}$ \\
\hline \hline \multirow{4}{*}{$\operatorname{Char}(\mathbb{F}) \neq 2,3$} & \multirow{2}{*}{$P_{1} \neq \pm P_{2}$} & $\begin{array}{l}\lambda=\frac{y_{2}-y_{1}}{x_{2}-x_{1}} \\
x_{3}=\lambda^{2}-x_{1}-x_{2} \\
y_{3}=\left(x_{1}-x_{3}\right) \lambda-y_{1}\end{array}$ \\
\cline { 2 - 3 } & \multirow{3}{*}{$\begin{array}{l}\lambda=\frac{3 x_{1}^{2}+a}{2 y_{1}} \\
x_{3}=\lambda^{2}-2 x_{1} \\
y_{3}=\left(x_{1}-x_{3}\right) \lambda-y_{1}\end{array}$} \\
\hline
\end{tabular}

TABLE I

ELLIPTIC CURVE POINT ADDITION $\left(P_{3}=P_{1}+P_{2}\right)$.

\begin{tabular}{|l||l|}
\hline $\operatorname{Char}(\mathbb{F})=2$ & $-P_{1}=\left(x_{1}, x_{1}+y_{1}\right)$ \\
\hline \hline $\operatorname{Char}(\mathbb{F}) \neq 2,3$ & $-P_{1}=\left(x_{1},-y_{1}\right)$ \\
\hline
\end{tabular}

TABLE II

ELLIPTIC CURVE POINT INVERSION.

\section{B. Point Multiplication}

Elliptic curve cryptographic techniques require the computation of point multiplication defined as repeated addition

$$
k P=\underbrace{P+P+\ldots+P}_{k \text { times }},
$$

where $P$ is an elliptic curve point, $k \in[1, n-1]$ is an integer, and $n$ is the order of the point $P$. To compute the point multiplication $k P$, the multiplier $k$ can be represented in base 2 as $k=\sum_{0 \leq i<s} k_{i} 2^{i}$, where $s$ is an integer typically close to $\left\lceil\log _{2} n\right\rceil, k_{i} \in B \cup\{0\}$ and $B$ is a set of nonzero integers including 1 . Then, Algorithm 1 can use this representation of $k$ to compute $k P$. Note that in the precomputation stage of this algorithm, we are only required to compute and store $d P$ for positive integers $d \in B$. This is because point inversion in $E(\mathbb{F})$ requires almost no computational effort. In the evaluation stage of this algorithm, we require about $s$ point doublings (it is in fact slightly less) and $(H(k)-1)$ point additions, where $H(k)$ denotes the Hamming weight of the representation of $k$.

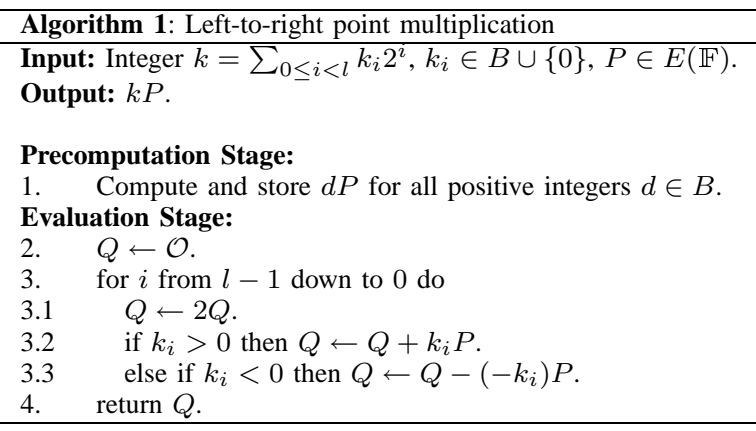

\section{The Proposed Representation}

Let $B=\left\{ \pm 1, \pm 3, \ldots, \pm\left(2^{w-1}-1\right)\right\}, l=\left\lceil\log _{2} n\right\rceil$, and $k=\sum_{0 \leq i<l} b_{i} 2^{i}, b_{i} \in\{0,1\}$ be the binary representation of an integer $k$. Then $k$ can be represented as $k=\sum_{0 \leq i \leq l} k_{i} 2^{i}$, $k_{i} \in B \cup\{0\}$ such that of any $w$ consecutive $k_{i}$ 's at most one is nonzero. This is a unique representation of $k$ called $w$-NAF. The length of the $w$-NAF of an integer is at most one digit longer than its binary representation, hence $k_{l}$ can be nonzero for $w$-NAF. It is also known that $w$-NAF has the minimum $H(k)$ among all radix-2 representations of $k$ with digits in $B$ [14]. This property makes $w$-NAF suitable for efficient implementation of point multiplication. However, the $w$-NAF recoding algorithm is a right-to-left algorithm, so we need to store the $w$-NAF representation of the multiplier before using it in a left-to-right point multiplication algorithm (such as Algorithm 1). In contrast to $w$-NAF, the proposed representation can be obtained using a left-to-right algorithm (Algorithm 2). In addition, in the proposed representation, we are able to use a more general set $B=\{ \pm 1, \pm 3, \ldots, \pm(2 m-$ $1)\}$, where $m$ is equal to the number of points we want to store in the precomputation stage. This feature allows us to use the available memory efficiently. For example, assume that we have enough memory to store three points. As a result, we can use the set $B=\{ \pm 1, \pm 3, \pm 5\}$. Let

$$
k=(10110111010101111110010110010001)_{2} .
$$

Using Algorithm 2, $k$ is recoded to

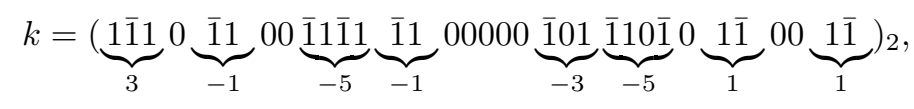

where $\overline{1}$ denotes -1 . Note that Algorithm 2 uses a binary signed-digit representation of $k$. This representation is in fact the output of the Booth recoding used in some multiplication and exponentiation algorithms [15], [16], [17]. 


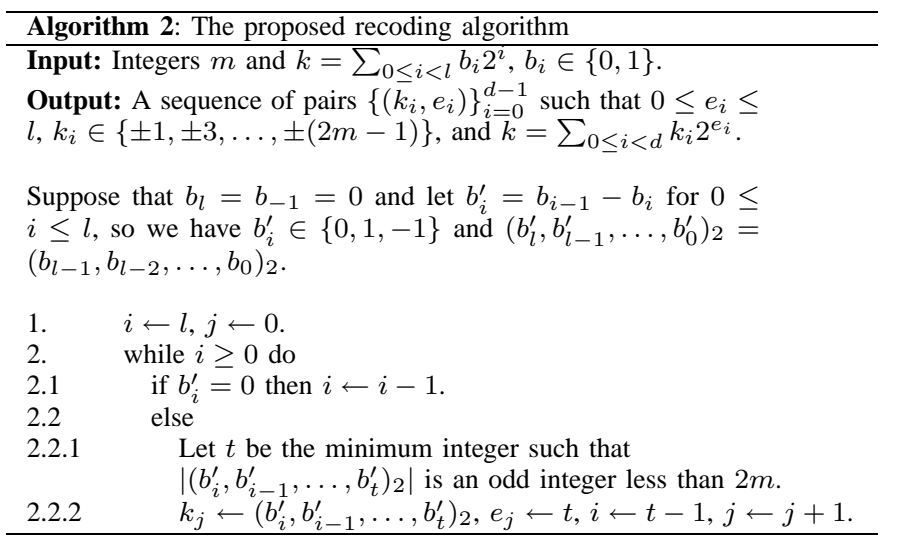

\section{AnAlysis of the Proposed Representation}

In this section we analyze the average Hamming weight of the proposed representation. To compare the average Hamming weight of the proposed representation with that of $w$-NAF, we use the same set $B$ as used for $w$-NAF, namely $B=$ $\left\{ \pm 1, \pm 3, \ldots, \pm\left(2^{w-1}-1\right)\right\}$. Let

$$
f(n)=\frac{1}{2^{n-1}} \sum_{2^{n-1} \leq N<2^{n}} H(N)
$$

and

$$
g(n)=\frac{1}{2^{n}} \sum_{0 \leq N<2^{n}} H(N) .
$$

$f(n)$ is the average Hamming weight of all $n$-bit numbers while $g(n)$ is the average Hamming weight of all $m$-bit numbers where $m \leq n$. From (4) and (5) we have

$$
\begin{aligned}
2^{n} g(n) & =\sum_{0 \leq N<2^{n}} H(N) \\
& =\sum_{0 \leq N<2^{n-1}} H(N)+\sum_{2^{n-1} \leq N<2^{n}} H(N) \\
& =2^{n-1} g(n-1)+2^{n-1} f(n) .
\end{aligned}
$$

Therefore, the two functions are related through the equation

$$
f(n)=2 g(n)-g(n-1) .
$$

Now suppose that $2^{n+w-2} \leq N<2^{n+w-1}$ is an integer. Therefore, $N$ can be represented as

$$
N=\sum_{0 \leq i<n+w-1} b_{i} 2^{i}, \quad b_{i} \in\{0,1\}
$$

and

$$
N=\sum_{0 \leq i<n+w} b_{i}^{\prime} 2^{i}, \quad b_{i}^{\prime} \in\{0,1,-1\}, b_{i}^{\prime}=b_{i-1}-b_{i} .
$$

These are the binary representation and the signed binary representation of $N$, respectively (note that to obtain the signed binary representation we assume that $\left.b_{n+w-1}=b_{-1}=0\right)$. We can also write $N$ uniquely as $N=q 2^{n}+r$ with $2^{w-2} \leq q<$ $2^{w-1}$ and $0 \leq r<2^{n}$. We will consider two cases, first when $b_{n-1}=0$ and then when $b_{n-1}=1$. For Case 1 we have

$\left(b_{n+w-1}^{\prime}, b_{n+w-2}^{\prime}, \ldots, b_{n}^{\prime}\right)_{2}=\left(b_{n+w-2}, b_{n+w-3}, \ldots b_{n}\right)_{2}=q$, and

$$
\left(b_{n-1}^{\prime}, b_{n-2}^{\prime}, \ldots, b_{0}^{\prime}\right)_{2}=\left(b_{n-1}, b_{n-2}, \ldots b_{0}\right)_{2}=r .
$$

Since $q \leq 2^{w-1}-1$, we deduce that $H(N)=1+H(r)$.

For Case 2 we have

$$
\begin{aligned}
\left(b_{n+w-1}^{\prime}, b_{n+w-2}^{\prime}, \ldots, b_{n}^{\prime}\right)_{2} & =\left(b_{n+w-2}, b_{n+w-3}, \ldots b_{n}\right)_{2}+1 \\
& =q+1
\end{aligned}
$$

and

$\left(b_{n-1}^{\prime}, b_{n-2}^{\prime}, \ldots, b_{0}^{\prime}\right)_{2}=\left(b_{n-1}, b_{n-2}, \ldots b_{0}\right)_{2}-2^{n}=r-2^{n}$.

Therefore, $H(N)=1+H\left(r-2^{n}\right)=1+H\left(2^{n}-r\right)$ (note that $H(M)=H(-M))$. These two cases can be summarized to

$$
H(N)= \begin{cases}1+H(r) & \text { if } 0 \leq r<2^{n-1} \\ 1+H\left(2^{n}-r\right) & \text { if } 2^{n-1} \leq r<2^{n}\end{cases}
$$

Then it follows by writing $N=q 2^{n}+r$ that

$$
\begin{aligned}
2^{n+w-2} f(n+w-1)= & \sum_{2^{n+w-2} \leq N<2^{n+w-1}} H(N) \\
= & 2^{w-2} \sum_{0 \leq r<2^{n-1}}(1+H(r)) \\
& +2^{w-2} \sum_{2^{n-1} \leq r<2^{n}}\left(1+H\left(2^{n}-r\right)\right) \\
= & 2^{w-2} \sum_{0 \leq r<2^{n-1}}(1+H(r)) \\
& +2^{w-2} \sum_{0<r \leq 2^{n-1}}(1+H(r)) \\
= & 2^{w-2}\left(2^{n-1} g(n-1)+2^{n-1}\right) \\
& +2^{w-2}\left(2^{n-1} g(n-1)+2^{n-1}+1\right) \\
= & 2^{n+w-2} g(n-1)+2^{n+w-2}+2^{w-2},
\end{aligned}
$$

so that

$$
f(n+w-1)=g(n-1)+1+2^{-n} .
$$

Combining (6) with (8), we obtain the recursion

$$
2 g(n+w)-g(n+w-1)-g(n)=1+2^{-(n+1)} .
$$

Clearly, we have

$$
H(N)= \begin{cases}0 & \text { if } N=0 \\ 1 & \text { if } 0<N<2^{w-1} \\ 1 & \text { if } 2^{w-1} \leq N<2^{w} \text { and } N \text { is even } \\ 2 & \text { if } 2^{w-1} \leq N<2^{w} \text { and } N \text { is odd }\end{cases}
$$

Using (5), we obtain

$$
\begin{aligned}
g(n) & =\frac{1}{2^{n}} \sum_{0 \leq N<2^{n}} H(N)=\frac{1}{2^{n}}\left(H(0)+\sum_{0<N<2^{n}} H(N)\right) \\
& =\frac{1}{2^{n}}\left(0+\left(2^{n}-1\right)\right)=1-2^{-n},
\end{aligned}
$$


when $1 \leq n \leq w-1$, and

$$
\begin{aligned}
g(n)= & \frac{1}{2^{w}} \sum_{0 \leq N<2^{w}} H(N) \\
= & \frac{1}{2^{w}}\left(H(0)+\sum_{\substack{0<N<2^{w-1}\\
}} H(N)\right. \\
& \left.+\sum_{\substack{2^{w-1} \leq N<2^{w} \\
N \text { even }}} H(N)+\sum_{\substack{w-1 \leq N<2 w \\
2^{w} \text { odd }}} H(N)\right) \\
= & \frac{1}{2^{w}}\left(0+\left(2^{w-1}-1\right)+2^{w-2}+2^{w-2} \times 2\right) \\
= & \frac{5}{4}-2^{-w},
\end{aligned}
$$

when $n=w$. Therefore, we get the initial conditions

$$
g(n)= \begin{cases}1-2^{-n} & \text { if } 1 \leq n \leq w-1 \\ \frac{5}{4}-2^{-w} & \text { if } n=w .\end{cases}
$$

Now let $u(n)=g(n)-\frac{n}{w+1}+2^{-(n+1)}$. It is easy to see that $u(n)$ satisfies the homogeneous recursion

$$
2 u(n+w)-u(n+w-1)-u(n)=0,
$$

with the initial conditions

$$
u(n)= \begin{cases}1-\frac{n}{w+1}-2^{-(n+1)} & \text { if } 1 \leq n \leq w-1 \\ \frac{1}{4}+\frac{1}{w+1}-2^{-(w+1)} & \text { if } n=w .\end{cases}
$$

To solve this recursion we use the following proposition from [18].

Proposition 1: Let $u(n)$ be a sequence satisfying the linear recursion $2 u(n+w)-u(n+w-1)-u(n)=0$ for $n \geq 1$. Then there exists $\rho>1$ such that as $n \rightarrow \infty$

$u(n)=\tau(n, w)=\frac{1}{w+1}\left(2 u(w)+\sum_{1 \leq i<w} u(i)\right)+O\left(\rho^{-n}\right)$.

Therefore, when $n \rightarrow \infty$ we can write

$$
\begin{aligned}
u(n)= & \frac{1}{w+1}\left(2\left(\frac{1}{4}+\frac{1}{w+1}-2^{-(w+1)}\right)+\sum_{1 \leq i<w} u(i)\right) \\
& +O\left(\rho^{-n}\right) \\
= & \frac{1}{w+1}\left(\left((w-1)-\frac{w(w-1)}{2(w+1)}-\left(\frac{1}{2}-2^{-w}\right)\right)\right. \\
& \left.+\left(\frac{1}{2}+\frac{2}{w+1}-2^{-w}\right)\right)+O\left(\rho^{-n}\right) \\
= & \frac{1}{w+1}\left(\frac{1}{w+1}+\frac{w}{2}\right)+O\left(\rho^{-n}\right) .
\end{aligned}
$$

Hence

$$
\begin{aligned}
g(n)= & u(n)+\frac{n}{w+1}-2^{-(n+1)} \\
= & \frac{n}{w+1}+\frac{1}{w+1}\left(\frac{1}{w+1}+\frac{w}{2}\right) \\
& +O\left(\rho^{-n}\right)-2^{-(n+1)}
\end{aligned}
$$

Applying (8) gives

$$
\begin{aligned}
f(n)= & g(n-w)+1+2^{-(n-w+1)} \\
= & \frac{n-w}{w+1}+\frac{1}{w+1}\left(\frac{1}{w+1}+\frac{w}{2}\right) \\
& +1-2^{-(n-w+1)}+2^{-(n-w+1)}+O\left(\rho^{-(n-w)}\right) \\
= & \frac{n}{w+1}-\frac{(w-1)(w+2)}{2(w+1)^{2}}+1+O\left(\rho^{-n}\right), \rho^{\prime}>1 .
\end{aligned}
$$

This is equal to the average Hamming weight of $w$-NAF when $n \rightarrow \infty$ [18]. (Note that in [18], the author computes the average number of group multiplications, which is equal to the average Hamming weight of $w$-NAF minus 1).

Similarly, we can obtain the average Hamming weight of the proposed representation for $B=\{ \pm 1, \pm 3, \ldots, \pm(2 m-1)\}$, where $2^{w-1} \leq 2 m<2^{w}$. Clearly (6) holds for this case too. However, instead of (7) we have

$$
H(N)= \begin{cases}1+H(r) & \text { if Case 1 } \\ 1+H\left(2^{n}-r\right) & \text { if Case 2 } \\ 1+H\left(2^{n-1}-r\right) & \text { if Case 3 } \\ 1+H(r) & \text { if Case 4 } \\ 1+H\left(r-2^{n-1}\right) & \text { if Case 5 } \\ 1+H\left(2^{n}-r\right) & \text { if Case 6 }\end{cases}
$$

where $2^{n+w-2} \leq N<2^{n+w-1}, N=q 2^{n}+r$ and

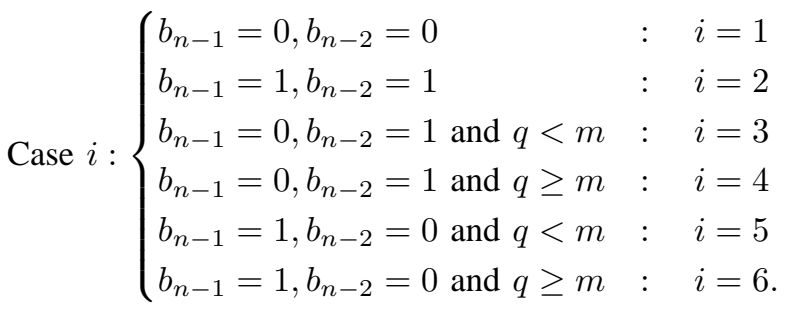

This is because

$$
\begin{aligned}
\left(b_{n+w-1}^{\prime}, b_{n+w-2}^{\prime}, \ldots, b_{n-1}^{\prime}\right)_{2}= & \left(b_{n+w-2}, b_{n+w-3}, \ldots, b_{n-1}\right)_{2} \\
& +b_{n-2} \\
= & 2 q+\left(b_{n-1}+b_{n-2}\right) .
\end{aligned}
$$

and

$$
\begin{aligned}
\left(b_{n-2}^{\prime}, \ldots, b_{0}^{\prime}\right)_{2} & =\left(b_{n-3}, b_{n-4}, \ldots, b_{0}\right)_{2}-b_{n-2} 2^{n-2} \\
& =\left(b_{n-1}, b_{n-2}, \ldots, b_{0}\right)_{2}-\left(b_{n-1}+b_{n-2}\right) 2^{n-1} \\
& =r-\left(b_{n-1}+b_{n-2}\right) 2^{n-1} .
\end{aligned}
$$

For example, for Case 5 we have

$$
\left(b_{n+w-1}^{\prime}, b_{n+w-2}^{\prime}, \ldots, b_{n-1}^{\prime}\right)_{2}=2 q+1 \leq 2 m-1,
$$

and

$$
\left(b_{n-2}^{\prime}, b_{n-3}^{\prime}, \ldots, b_{0}^{\prime}\right)_{2}=r-2^{n-1} .
$$

Therefore, $H(N)=1+H\left(r-2^{n-1}\right)$. Using (4) and (9) it follows that 


$$
\begin{array}{rl}
2^{n+w-2} & f(n+w-1)=\sum_{1 \leq i \leq 6 \text { Case } i} \sum_{(N)} H(1+H(r)) \\
= & 2^{w-2} \sum_{0 \leq r<2^{n-2}}\left(1+\sum_{0<r \leq 2^{n-2}}(1+H(r))\right. \\
& +2^{w-2} \sum_{0<r \leq 2^{n-2}}(1+H(r)) \\
& +\left(m-2^{w-2}\right) \sum_{0 \leq r<2^{n-2}}(1+H(r)) \\
& +\left(2^{w-1}-m\right) \sum_{2^{n-2} \leq r<2^{n-1}}(1+H(r)) \\
& +\left(m-2^{w-2}\right) \sum_{0}(1+H(r)) \\
& +\left(2^{w-1}-m\right) \sum_{2^{n-2}<r \leq 2^{n-1}}\left(2^{w-1}-m\right) 2^{n} g(n-1)+\left(m-2^{w-2}\right) 2^{n} g(n-2) \\
& +2^{n+w-2}+m .
\end{array}
$$

so that

$$
f(n+w-1)=\alpha g(n-1)+\beta g(n-2)+1+(\beta+1) 2^{-n},
$$

where $\alpha=\frac{2^{w-1}-m}{2^{w-2}}, \beta=\frac{m-2^{w-2}}{2^{w-2}}$ and $\alpha+\beta=1$. Combining (6) with (10), we get the recursion

$2 g(n+w)-g(n+w-1)-\alpha g(n)-\beta g(n-1)=1+(\beta+1) 2^{-(n+1)}$

Setting $u(n)=g(n)-\frac{n}{w+\beta+1}+2^{-(n+1)}$ gives the homogeneous recursion

$$
2 u(n+w)-u(n+w-1)-\alpha u(n)-\beta u(n-1)=0 .
$$

To solve this recursion we can use the following proposition.

Proposition 2: Let $\mathrm{u}(\mathrm{n})$ be a sequence satisfying the linear recursion $2 u(n+w)-u(n+w-1)-\alpha u(n)-\beta u(n-1)=0$ for $n>1$ and $\alpha+\beta=1$. Then there exists $\rho>1$ such that as $n \rightarrow \infty$

$$
\begin{aligned}
u(n)= & \alpha \tau(n, w)+\beta \tau(n-1, w+1) \\
= & \frac{\alpha}{w+1}\left(2 u(w)+\sum_{1 \leq i<w} u(i)\right) \\
& +\frac{\beta}{w+2}\left(2 u(w+1)+\sum_{1 \leq i<w+1} u(i)\right)+O\left(\rho^{-n}\right) .
\end{aligned}
$$

Using this proposition we get

$$
\begin{aligned}
g(n)= & u(n)+\left(\frac{n}{w+\beta+1}-2^{-(n+1)}\right) \\
= & \left(\frac{n}{w+\beta+1}-2^{-(n+1)}\right) \\
& +\frac{\alpha}{w+1}\left(\frac{w}{2}-\frac{\beta}{2}+\frac{\beta w+2 \beta+2}{2(w+\beta+1)}\right) \\
& +\frac{\beta}{w+2}\left(\frac{w}{2}+\frac{3}{4} \alpha+\frac{\beta(w+4)}{2(w+\beta+1)}\right)+O\left(\rho^{-n}\right) \\
= & \frac{n}{w+\beta+1}-2^{-(n+1)}+\gamma+O\left(\rho^{-n}\right),
\end{aligned}
$$

where

$$
\begin{aligned}
\gamma= & \frac{\alpha}{w+1}\left(\frac{w}{2}-\frac{\beta}{2}+\frac{\beta w+2 \beta+2}{2(w+\beta+1)}\right) \\
& +\frac{\beta}{w+2}\left(\frac{w}{2}+\frac{3}{4} \alpha+\frac{\beta(w+4)}{2(w+\beta+1)}\right) \\
\approx & \frac{1}{2} .
\end{aligned}
$$

Finally, applying (6) gives

$$
\begin{aligned}
f(n)= & 2 g(n)-g(n-1) \\
= & 2\left(\frac{n}{w+\beta+1}-2^{-(n+1)}+\gamma\right) \\
& -\left(\frac{n-1}{w+\beta+1}-2^{-n}+\gamma\right) \\
= & \frac{n+1}{w+\beta+1}+\gamma .
\end{aligned}
$$

\section{Performance Evaluation and Comparison with PreVious AlgorithMS}

In this section, we first obtain the memory requirements of the proposed recoding algorithm (Algorithm 2). We explain the advantages of the proposed algorithm over the previous ones and summarize them in a table.

In Algorithm 2, the operation $b_{i}^{\prime}=b_{i-1}-b_{i}$ is only used to explain the recoding process. In other words, the computation of $b_{i}^{\prime}$ is not required for the implementation of this algorithm. To implement Algorithm 2, the condition $b_{i}^{\prime}=0$ in Step 2.1 can be replaced with the equivalent condition $b_{i}=b_{i-1}$. We can also compute $\left(b_{i}^{\prime}, b_{i-1}^{\prime}, \ldots, b_{t}^{\prime}\right)_{2}$ in Steps 2.2.1 and 2.2.2 using the following equation

$$
\left(b_{i}^{\prime}, b_{i-1}^{\prime}, \ldots, b_{t}^{\prime}\right)_{2}=\left(b_{i-1}, \ldots, b_{t}\right)_{2}-b_{i} 2^{i-t}+b_{t-1} .
$$

Let $2^{N-1} \leq 2 m<2^{N}$ and $A_{i, j}=\left(b_{i}^{\prime}, b_{i-1}^{\prime}, \ldots, b_{j}^{\prime}\right)_{2}$. In Steps 2.2.1 and 2.2.2 we have $b_{i}^{\prime} \neq 0$, thus from (11) we get

$$
2^{i-j-1} \leq\left|A_{i, j}\right| \leq 2^{i-j} .
$$

Set

$$
T= \begin{cases}0 & \text { if } i-N<0 \\ i-N & \text { if }\left|A_{i, i-N}\right| \leq 2 m \\ i-N+1 & \text { otherwise }\end{cases}
$$


Using (12), it is easy to prove that $T$ is the minimum integer such that $\left|A_{i, T}\right| \leq 2 m$. Suppose $A_{i, T}=2^{r} V$, where $r \geq 0$ and $V$ is an odd integer. From $A_{i, j}=2 A_{i, j-1}+b_{j}^{\prime}$ we have $A_{i, j-1}=A_{i, j} / 2$ when $A_{i, j}$ is even. Therefore, $V=A_{i, T-r}$ and $(t=T-r)$ is the minimum integer such that $\left|A_{i, t}\right|$ is an odd integer less than $2 m$. Consequently, $t$ and $k_{j}$ in Steps 2.2.1 and 2.2.2 of Algorithm 2 can be simply obtained by first computing $T$ and $A_{i, T}$ using (11) and (13), and then shifting $A_{i, T}$ to the right (dividing it by two), until an odd integer is obtained. From (13), we can see that the length of $A_{i, T}$ is at most $N$. Therefore, Algorithm 2 requires $O(N)$ (or $\left.O\left(\log _{2}(m)\right)\right)$ bits of memory to generate $A_{i, t}$.

Right-to-left recoding algorithms require $O(l)$ bits of memory because they need to store the recoded integer. Consequently, the memory requirements for generating the proposed representation is less than the memory requirements for generating $w$-NAF and the proposed representation in [10]. In [11], the authors propose an optimal left-to-right binary signeddigit recoding. However, this representation uses a specific set $B=\{ \pm 1\}$. The representation proposed in this paper uses a general set $B=\{ \pm 1, \pm 3, \ldots, \pm(2 m-1)\}$. Therefore it uses memory more efficiently compared to $w$-NAF, the optimal binary signed-digit representation [11] and the representation proposed in [12]. For example, if we have sufficient memory to store $\left(2^{t}-1\right)$ points, using $w$-NAF and the representation proposed in [12] we can store up to $2^{(t-1)}$ points (about $50 \%$ of available memory), and the rest of the memory is unused.

Table III summarizes characteristics of the proposed representation and the previous ones. In the second column, $C$ indicates the maximum number of points that can be stored. The proposed representation can use all the available memory by setting $m=C$. In this case, the average Hamming weight of the proposed representation is $\bar{H}(N)=\frac{l}{w+\beta+1}$, where $\beta=\left(\frac{C}{2^{2 \log _{2} C J}}-1\right)$ and $w=\left\lfloor\log _{2} C\right\rfloor+2$. The last column indicates the extra memory required by the recoding algorithms. As shown in this table, the proposed recoding algorithm requires a small amount of extra memory. It also generates a minimal average Hamming weight representation and does not waste memory.

\begin{tabular}{|l|c|c|c|}
\hline representation & wasted Mem. & $H(N)$ & extra Mem. \\
\hline \hline$w$-NAF & $0 \%-50 \%$ & $\frac{l}{w+1}$ & $O(l)$ \\
\hline Binary signed-digit [11] & $100\left(1-\frac{1}{C}\right) \%$ & $\frac{l}{3}$ & $O(1)$ \\
\hline Fractional windows [10] & $0 \%$ & $\frac{l}{w+\beta+1}$ & $O(l)$ \\
\hline The Prop. Rep. in [12] & $0 \%-50 \%$ & $\frac{l}{w+1}$ & $O\left(\log _{2}(m)\right)$ \\
\hline Our Prop. Rep., Sec. III & $0 \%$ & $\frac{l}{w+\beta+1}$ & $O\left(\log _{2}(m)\right)$ \\
\hline
\end{tabular}

TABLE III

COMPARISON OF CHARACTERISTICS OF THE PROPOSED AND PREVIOUS REPRESENTATIONS.

\section{ApPLICATIONS}

In Section IV we showed that the proposed representation has minimal average Hamming weight. Minimal representations have applications in the theory of arithmetic codes [19], in fast arithmetic techniques [20] and in fast point multiplication algorithms [7]. In this section, we consider the proposed representation in fast point multiplication algorithms.

Algorithms 3 and 4 are two typical point multiplication algorithms. In Algorithm 3 the digits are scanned from left-toright while in Algorithm 4 they are scanned from right-to-left. In practice, the left-to-right algorithm (Algorithm 3) is preferable. This is because Algorithm 3 can be significantly sped up by precomputing $d P$ for positive integers $d \in B$. Furthermore, Algorithm 4 requires extra memory for storing the point $R$.
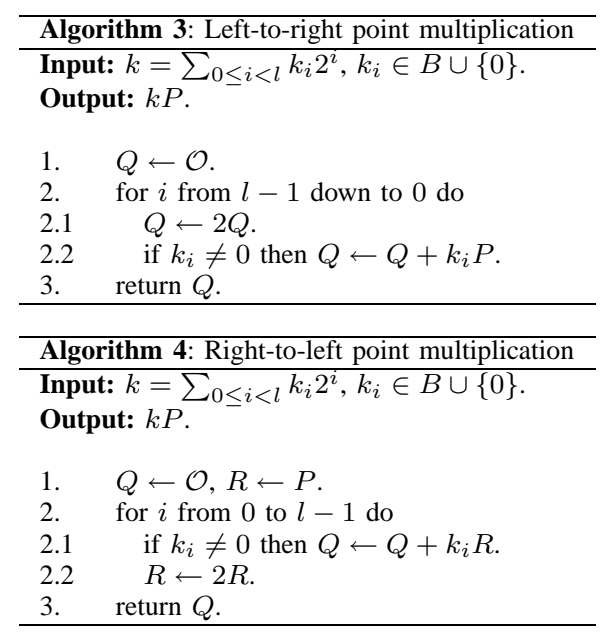

The integer representation of the multiplier plays an important role in the performance of Algorithm 3. For example, the size of the set $B$ and the Hamming weight of the integer representation determine the number of point additions required in Algorithm 3. This is why we are interested in integer representations with minimal average Hamming weight. We are also interested in left-to-right recoding algorithms (such as the proposed recoding algorithm) as they enable us to perform both recoding and point multiplication simultaneously. Note that to compute $k P$ using Algorithm 3 , the multiplier $k$ must be recoded and stored in advance if a right-to-left recoding algorithm is used. In this case, we require extra memory $O(l)$ which is undesirable for constraint devices such as smart cards. For these devices, it is also important to use the available memory efficiently. As the proposed representation uses a general set $B$, it can satisfy this requirement too. Algorithm 5 , shows how the proposed representation can be used in a left-to-right point multiplication algorithm. Note that we can use a direct $2^{r} P$ computation algorithm [21], [22] to compute $2^{i-t+1} Q$ in line 3.3 of Algorithm 5 to further improve the speed of this algorithm. 


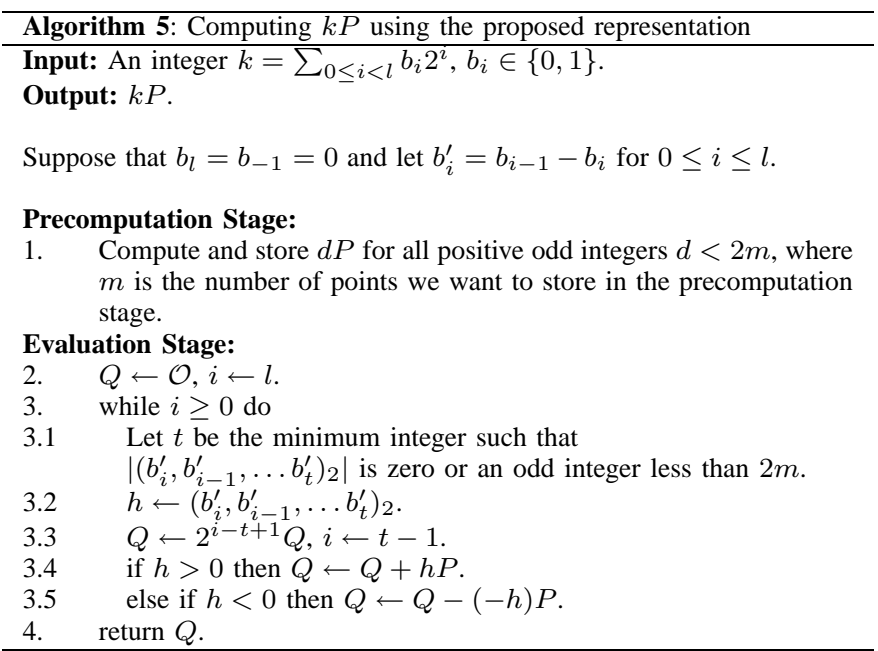

\section{CONCLUSION}

In this paper, a new radix-2 representation was proposed. The average Hamming weight of the proposed representation was analyzed, and shown to be equal to the average Hamming weight of $w$-NAF. This is an important property, as it speeds up point multiplication methods by reducing the required number of point additions/subtractions.

The proposed representation has two other properties which are especially attractive for efficient implementation of point multiplication methods in devices with a small amount of memory. First, the proposed representation can be obtained by scanning the bits from left to right. Consequently, there is no need to store the recoded representation in advance, and hence the memory requirements are reduced. Second, using the proposed representation, we can store as many points as we want (up to the limits of available memory). Therefore, we can use memory efficiently to improve performance.

\section{REFERENCES}

[1] N. Koblitz, "Elliptic curve cryptosysmtems," Math. Comp., vol. 48, pp. 203-209, 1987.

[2] V. Miller, "Uses of elliptic curves in cryptography," Springer-Verlag Lecture Notes in Computer Science, vol. 218, pp. 417-426, 1987.

[3] W. Diffie and M. E. Hellman, "New directions in cryptography," IEEE Trans. Inform. Theory, vol. 22, pp. 644-654, 1976.

[4] T. ElGamal, "A public key cryptosystem and a signature scheme based on the discrete logarithm," IEEE Trans. Inform. Theory, vol. 31, pp. 469-472, 1985.

[5] I. Blake, G. Seroussy, and N. Smart, Elliptic Curves in Cryptography. Cambridge: Cambridge University Press, 1999.

[6] A. Menezes, P. van Oorschot, and S. Vanstone, Handbook of Applied Cryptography. CRC Press, 1997.

[7] D. Gordon, "A survay of fast exponentiation methods," J. Algorithms, vol. 27, pp. 129-146, 1998

[8] V. Müller, "Fast multiplication on elliptic curves over small fields of characteristic two," J. Crypt., vol. 11, pp. 219-234, 1998.

[9] J. A. Solinas, "Efficient arithmetic on Koblitz curves," Designs, Codes and Cryptography, vol. 19, pp. 195-249, 2000.

[10] B. Möller, "Improved techniques for fast exponentiation," SpringerVerlag Lecture Notes in Computer Science, vol. 2587, pp. 298-312, 2003.

[11] M. Joye and S. M. Yen, "Optimal left-to-right binary signed-digit recoding," IEEE Trans. Comput., vol. 49, pp. 740-748, 2000.

[12] J. A. Muir and D. R. Stinson, "New minimal weight representations for left-to-right window methods," Preprint, 2004, Available from http://www.cacr.math.uwaterloo.ca/tech_reports.html.
[13] K. Okeya, K. Schmidt-Samoa, C. Spahn and T. Takagi, "Signed binary representations revisited," Springer-Verlag Lecture Notes in Computer Science, vol. 3152, pp. 123-139, 2004.

[14] J. A. Muir and D. R. Stinson, "Minimality and other properties of the with- $w$ nonadjacent form," Preprint, 2004, Available from http://www.cacr.math.uwaterloo.ca/tech_reports.html.

[15] K. Hwang, Computer Arithmetic, Principles, Architecture, and Design. New York: John Wiley \& Sons, 1989.

[16] R. S. Katti, "Speeding up elliptic cryptosystems using a new signed binary representation for integers," Proc. Euro-Micro Conf. Digital Design, 2002.

[17] C. K. Koc, "Parallel canonical recoding," Electronics Letters, vol. 32, pp. 2063-2065, 1996.

[18] H. Cohen, "Analysis of the flexible window powering algorithm," Preprint, 2004, Available from http://www.math.ubordeaux.fr/ $/$ cohen/window.dvi.

[19] J. H. van Lint, Introduction to Coding Theory. Springer-Verlag, 1982.

[20] A. D. Booth, "A signed binary multiplication technique," The Quaterly Journal of Mechanics and Applied Mathematics, vol. 4, pp. 236-240, 1951, reprinted in [Swa90, vol. I, pp. 100-104].

[21] J. Guajardo and C. Paar, "Efficient algorithms for elliptic curve cryptosystems," Springer-Verlag Lecture Notes in Computer Science, vol. 1294, pp. 342-356, 1997.

[22] Y. Sakai and K. Sakurai, "Efficient scalar multiplications on elliptic curves with direct computations of several doublings," IEICE Trans. Fundamentals, vol. E84-A, pp. 120-129, 2001. 